\title{
Improving mid stream urine sampling: reducing labelling error and laboratory rejection
}

\author{
Adam Jakes, Eleanor McCue, Alison Cracknell \\ Leeds Teaching Hospitals NHS Trust, UK
}

\begin{abstract}
A urine sample is vital in older patients with pyrexia or acute confusion, and commonly directs clinicians towards a source of infection. Not only can the organism be identified, but sensitivities to antibiotics can also guide prescribing. A high number of urine samples were not being processed on the medicine for older people wards at St. James's Hospital due to incomplete hand-written request forms not complying with trust policy. Previous attempts to re-educate staff had failed to improve acceptance rates. Rejected samples delay diagnosis, identification of organisms and subsequent sensitivities, as well as increasing staff workload.
\end{abstract}

A total of 72 urine samples were audited from our wards in March 2013; 12 (17\%) rejected. Clinicians were notified of rejected samples within one to four days. An electronic-requesting system was implemented in April 2013. Once implemented, a further two data collection cycles of 72 urine samples were completed from the same wards. In December 2013, 55 (76\%) were electronically requested and 17 (24\%) handwritten. Four (5\%) samples were rejected and were all hand-written. In August 2014, 61 (85\%) were electronically requested and 11 (15\%) hand-written. No samples were rejected.

The electronic-requesting system has effectively reduced the number of rejected urine samples. No electronically requested samples were rejected, therefore $100 \%$ sample acceptance is achievable. It is more effective than re-educating staff alone and ensures requests meet trust policy. Clinicians were notified of a samples rejection after one to four days. By this time patients may have started antibiotic therapy, decreasing the likelihood of isolating the causative organism in subsequent samples.

All urine samples requested must meet a high standard and comply with trust policy in order to be processed. An electronic-requesting system removes errors of omission and ensures policy compliance, ultimately leading to improved patient care. Now our processes are reliable we will go onto measure changes at patient level, e.g. confirmed diagnoses of urine infection, outcomes of earlier narrow spectrum antibiotics, and length of stay.

\section{Problem}

It had been noted that a high number of mid-stream urine samples were being rejected by the microbiology laboratory on the medicine for older people wards at St. James's Hospital. This was due to incomplete request forms not complying with the Leeds Teaching Hospital NHS Trust urine sample policy. Rejected samples delay diagnosis, identification of organisms and subsequent sensitivities, together with increasing staff workload. Previous attempts to reeducate staff have failed to improve acceptance rates. There is a process where rejected samples can be released by sending an additional form with the required information to the laboratory, however this is time-consuming and can take a considerable amount of time.

The Leeds Teaching Hospital NHS Trust decided to implement an electronic-requesting system (Ordercomms), which allows users to print out pre-completed request forms (including mid-stream urine samples). Both electronic and written request forms would continue to be accepted, however staff were encouraged to use the electronic forms.

\section{Background}

A mid-stream urine sample is vital in older patients with pyrexia or acute confusion, and commonly directs clinicians towards a source of infection. Not only can the organism be identified, but sensitivities to antibiotics can also guide prescribing.

The Leeds Teaching Hospital NHS Trust policy on mid-stream urine sample labelling is that all samples must contain the patient's full name, date of birth, and hospital/NHS number. There must also be an indication for the sample being taken, together with a healthcare professional's name and contact details. Failure to comply with this policy will result in the sample being rejected from the laboratory and not processed until further information is received.

Electronic-requesting systems have been shown to increase the accuracy and completeness of information when compared to a paper-based system.[1, 2]

\section{Baseline measurement}

An audit was designed to assess whether the rejection of mid- 
stream urine samples was an isolated issue or whether it was happening on all medicine for older people wards. The number of rejected mid-stream urine samples was assessed over two weeks.

All mid-stream urine samples sent from the medicine for older people wards at St. James's Hospital were audited. The hospital result server was used to highlight and collect data on urine samples that have been processed or rejected. Patient demographic data along with the date of sample collection and laboratory result were recorded. The date of result authorisation was noted so that the time to diagnosis/notification of rejection could be calculated. All data was anonymised and kept in a password-protected computer within a locked facility.

A total of 72 mid-stream urine samples from 44 patients were sent to the microbiology laboratory from the medicine for older people wards between 1st March and 15th March 2013. Twelve (17\%) samples were rejected, with only three being overridden, and the remaining nine samples presumably discarded. The time taken for the 12 samples to be authorised and rejected ranged from one to four days.

The reasons for rejection were:

- No clinical details (seven)

- No healthcare professional details (four)

- Multiple breaches of protocol (one)

Of the 60 processed urine samples, $35(58 \%)$ were positive and 25 $(42 \%)$ were negative. The average wait for results to be authorised was two days (minimum one day, maximum five days). One month after the audit was conducted, an electronic-requesting system (Ordercomms) was implemented allowing users to print out precompleted mid-stream urine order forms.

See supplementary file: ds3817.png - "Hand written request form"

\section{Design}

A second and third data collection cycle was designed to assess whether the new electronic-requesting system had reduced the number of rejected mid-stream urine samples.

A further 72 mid-stream urine samples sent from the medicine for older people wards at St James's Hospital were audited in December 2013 and August 2014. The hospital results server was used to identify urine samples that had been processed or rejected. Patient demographic data along with the date of sample collection and laboratory result was recorded. The date of results authorisation was noted so that the time to diagnosis/notification of rejection could be calculated. Whether the urine samples were processed using electronically printed order forms or hand-written forms was also noted. This was possible as samples ordered through the electronic-requesting system are given a unique reference number.

\section{Strategy}

The electronic-requesting system (Ordercomms) was implemented in April 2013, allowing users to print out pre-completed mid-stream urine order forms. It was first trialled in the acute medical unit and medicine for older people wards, before being rolled out to all of the medical wards. Nominated information technology staff provided training and drop-in sessions were arranged. Staff were encouraged to use this system rather than hand writing the order forms. In July 2013, phlebotomists were instructed not to routinely accept hand-written requests on their morning rounds.

\section{Results}

December 2013:

A further 72 mid-stream urine samples were identified; 55 (76\%) were requested using the electronic-requesting system and 17 (24\%) were hand-written. Four (5\%) samples were rejected and were all hand-written. None of the rejected samples were overridden, however three were subsequently repeated using the electronic-requesting system and accepted by the laboratory. The time taken for the four rejected samples to be authorised ranged from one to three days.

The reasons for rejection were:

- Multiple breaches of protocol (two)

- The urine specimen and order form did not have two matching identifiers (one)

- Specimen container was empty (one)

Of the 68 processed urine samples, 18 (25\%) were positive and 50 $(70 \%)$ were negative. The average wait for results to be authorised was two days (minimum one day, maximum four days).

August 2014:

A further 72 mid-stream urine samples were identified; 61 (85\%) were requested using the electronic-requesting system, and 11 $(15 \%)$ were hand-written. No mid-stream urine samples were rejected.

Of the 72 processed urine samples; 24 (33\%) were positive and 48 $(67 \%)$ were negative. The average wait for results to be authorised was two days (minimum one day, maximum four days).

See supplementary file: ds3818.png - "Electronic requesting form"

\section{Lessons and limitations}

An electronic-requesting system is more effective than re-educating staff alone and ensures requests meet trust policy.

A limitation of the audit was that samples could still be rejected as hand-written request forms continued to be sent. These request forms could not be completely removed; as with any electronicrequesting system there needs to be a back-up method of requesting urine samples if the system fails. If a hand-written 
request is to be used, then the healthcare professional should ensure all details are accurate and complete.

\section{Conclusion}

All urine samples requested must meet a high standard and comply with trust policy in order to be processed. An electronic-requesting system removes errors of omission and ensures policy compliance; ultimately leading to improved patient care. Now our processes are reliable we will go onto measure changes at patient level, e.g. confirmed diagnoses of urine infection, outcomes of earlier narrow spectrum antibiotics, and length of stay.

\section{References}

1. Sittig DF, Stead WW. Computer-Based Physician Order Entry: The State of the Art. J Am Med Inform Assoc. 1994 Mar-Apr; 1(2): 108-23.

2. Poissant L, Pereira J, Tamblyn R, Kawasumi Y. The Impact of Electronic Health Records on Time Efficiency of Physicians and Nurses: A Systematic Review. J Am Med Inform Assoc. 2005 Sep-Oct; 12(5): 505-16.

\section{Declaration of interests}

Nothing to declare.

\section{Acknowledgements}

The results were presented at the British Society of Microbial Technology meeting 2014. 